\title{
The Acute Effect of Static Stretching Applied to Different Muscle Groups of the Lower Extremity on Sprint Running
}

\author{
Zeliha Başkurt ${ }^{1}$, Sabriye Ercan ${ }^{2}$, Ferdi Başkurt ${ }^{3}$, Tuba İnce Parpucu ${ }^{4}$ \\ ${ }^{I}$ (Physiotherapy and Rehabilitation Department, Suleyman Demirel University, Health Sciences Faculty, \\ Turkey) \\ ${ }^{2}$ (Department of Sports Medicine, Dr.Ersin Arslan Education and Research Hospital, Turkey) \\ ${ }_{3}^{3}$ (Physiotherapy and Rehabilitation Department, Suleyman Demirel University, Health Sciences Faculty, \\ Turkey) \\ ${ }^{4}$ (Physiotherapy and Rehabilitation Department, Suleyman Demirel University, Health Sciences Faculty, \\ Turkey)
}

\begin{abstract}
Stretching is a conventional method applied by athletes to reach the maximum performance at the warm-up stage and to protect them from injuries. Although it is frequently applied by athletes, there is no consensus on the acute effects of stretching exercises on the performance. The aim of this study is to examine the acute effect of static stretching applied to quadriceps and/or hamstring muscle groups on sprint running.

45 males (average $( \pm S D)$ age $22,9( \pm 1,3)$ years; height $178,1( \pm 6,4) \mathrm{cm}$; body weight $77,7( \pm 12) \mathrm{kg}$ ) participated in the study. Following a 5-minute of submaximal warm-up run, 3 different stretching protocols (Method I: only 5-minute warm-up run; Method II: 5- minute warm-up run and static hamstring stretching; Method III: 5- minute warm-up run, static hamstring and Quadriceps femoris stretching) were applied to the participants by being randomized on non-consecutive days. A 20-meter sprint test was conducted after the stretching protocols. At the end of the study, it was identified that passive stretching applied to the hamstring muscle had a numerically positive effect on the acute sprint performance, however, there was no statistically significant difference between Method I, II, III ( $p>0.05$ ). It was observed that static stretching exercises applied to different muscle groups of the lower extremity did not have a negative effect on the 20-meter sprint time performance. Therefore, we think that short-term static stretching exercises should be performed before the competition, without the concern of performance loss.
\end{abstract}

Keywords: hamstring, quadriceps, sprint time, static stretching.

\section{Introduction}

All the preparations made before the competition are aimed at adapting the athlete to the competition. A well-designed warm-up period helps athletes to get ready for the competition mentally and physically [1]. A classic warm-up period consists of submaximal running, static stretching exercises applied to big muscle groups and joint range of motion exercises [1]. Low-medium intensity aerobic exercises increase blood flow, internal heat of muscles and the body [1,2]. The increase in the internal heat of muscles has a critical role in providing effective results in short-term performances [1]. Joint range of motion exercises increase neural transmissions and facilitate the power/speed requiring situations [1]. Stretching is a conventional method applied by athletes to reach the maximum performance at the warm-up stage and to protect them from injuries [3]. The three most important benefits of stretching are that it decreases the risk of injury, the muscle fatigue occurring after exercising is rapidly relieved and athletic performance is affected [4]. Although it is frequently applied by athletes, there is no consensus on the acute effects of stretching exercises on the performance [3]. When the literature is examined, there is no conclusive evidence of which stretching type is the best, and it is found that different stretching types at different dosages provide different results on the acute performance [3].

Stretching exercises consist of ballistic, dynamic, static and proprioceptive neuromuscular facilitation stretching [5]. While ballistic stretching is performed by forcing the normal joint range of motion of the body, dynamic stretching is done by using the body's own weight at the limits of normal joint motion $[5,6]$. Proprioceptive neuromuscular facilitation stretching is a special type of stretching including both stretching and contraction components [5,7]. On the other hand, static stretching is a reliable type of stretching that is most commonly used and involves the lowest risk of injury, is performed without creating motion at the final point after the muscle stretches to a certain point [6,7].

It is known that static stretching increases the joint range of motion acutely and protects from injuries [8]. However, according to the information obtained from various studies, while there are authors arguing that it increases the sprint time, skip distance, and muscle strength, there are also authors who argue that it decreases the performance $[1,8]$. Those who argue that static stretching affects the performance of athletes negatively state that the increase in the musculotendinous unit stiffness and neural inhibition decrease the power output [2]. 
Moreover, the fact that stretching applied to the single extremity affects bilateral extremity indicates that neural inhibition results from mechanisms of not only local but also central nervous system [9]. On the other hand, those arguing that stretching affects the performance positively state that a stretched hamstring muscle prevents reaching the maximum speed during the sprint and the muscle flexibility should be provided with static stretching exercises [10].

The presence of opposing views on stretching exercises brings the questions of when, how often and what type of stretching athletes should apply to which muscle groups [8]. It was identified that the current studies on the acute effect of stretching exercise on performance focus on stretching types and stretching times. Although a study examining the effect of unilateral stretching exercise has been encountered in the literature review we conducted, there is no study examining the effect of static stretching applied to different muscle groups on sprint running. The aim of this study is to examine the acute effect of static stretching applied to quadriceps and/or hamstring muscle groups on sprint running.

\section{Material And Method}

45 males studying at Süleyman Demirel University, Faculty of Health Sciences participated in the study on a voluntary basis. The participants had a reactive physical activity. There was no lower extremity injury and surgery in the history of the participants. No shortness of the lower extremity muscles, acute musculoskeletal system injury, cardiovascular and pulmonary system pathology that would prevent exercising were identified during the physical examination. All participants were informed about not performing highintensity exercises, not using any drugs or drinking beverages containing alcohol and caffeine within 24 hours before the day of the test. The participants ate their last meal at least 2 hours before the tests.

Prior to the study, all participants were informed about all kinds of benefits and risks of the study. The study was approved by Suleyman Demirel University, Local Ethics Committee. The study was conducted in accordance with the "Declaration of Helsinki".

\subsection{Protocols}

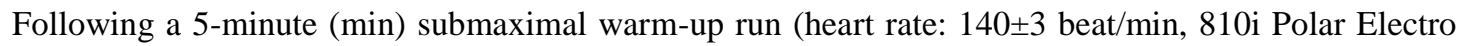
Inc., Finland), 3 different stretching protocols (Method I: only 5-min warm-up run; Method II: 5-min warm-up run and static hamstring stretching; Method III: 5-min warm-up run, static hamstring and Quadriceps femoris (QF) stretching) were applied to all of the participants by being randomized on non-consecutive days to evaluate the effect of static stretching applied to different muscle groups in the lower extremity (Table 1).

Table 1: Randomization of stretching protocols (n:45)

\begin{tabular}{|c|c|c|c|c|c|c|}
\hline & \multirow{4}{*}{ 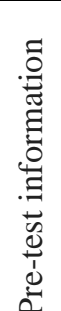 } & 1 st day & \multirow[b]{4}{*}{$\overrightarrow{\tilde{Q}}$} & 3rd day & \multirow[b]{4}{*}{$\overrightarrow{\tilde{Q}}$} & 5th day \\
\hline Group A [n:15] & & Method I & & Method II & & Method III \\
\hline Group B [n:15] & & Method II & & Method III & & Method I \\
\hline Group C [n:15] & & Method III & & Method I & & Method II \\
\hline
\end{tabular}

Static stretching exercises were applied to hamstring and/or QF muscle groups in 10 repetitions by the method recommended by Alter [11,12]. The participants sat down on the floor in the position in which both lower extremities were at full extension for hamstring static stretching (\#46). Then, they extended towards the feet with their bodies. Meanwhile, attention was paid to keeping their backs straight. When tension was felt in the hamstring region, this position was maintained for 15 seconds (sec). On the other hand, for QF static stretching, knee flexion was achieved by grasping with the hand above the ankle or from the forefoot. The participants were asked to move their heels towards the hip. When tension was felt in the QF region, this position was maintained for $15 \mathrm{sec}$. During the process, the participants were allowed to get support from the wall to ensure balance. Stretching exercises were applied to the bilateral extremity.

Following the stretching protocols, it was proceeded to 20-meter (m) sprint running (Figure 1). The indoor running track was preferred to reduce the effect of the environmental factors on the sprint performance to the lowest level. The 20-m running test was used to identify the effect of static stretching applied to different muscle groups on the acute sprint performance [2]. At the start of the run, the sprint preparation position, in which the hands were in contact with the ground and a leg was behind, was selected. The photocell device with a chronometer was used to determine the sprint times. At the end of $20 \mathrm{~m}$, the sprint time determined with a photocell was recorded in separate forms for each process day. 


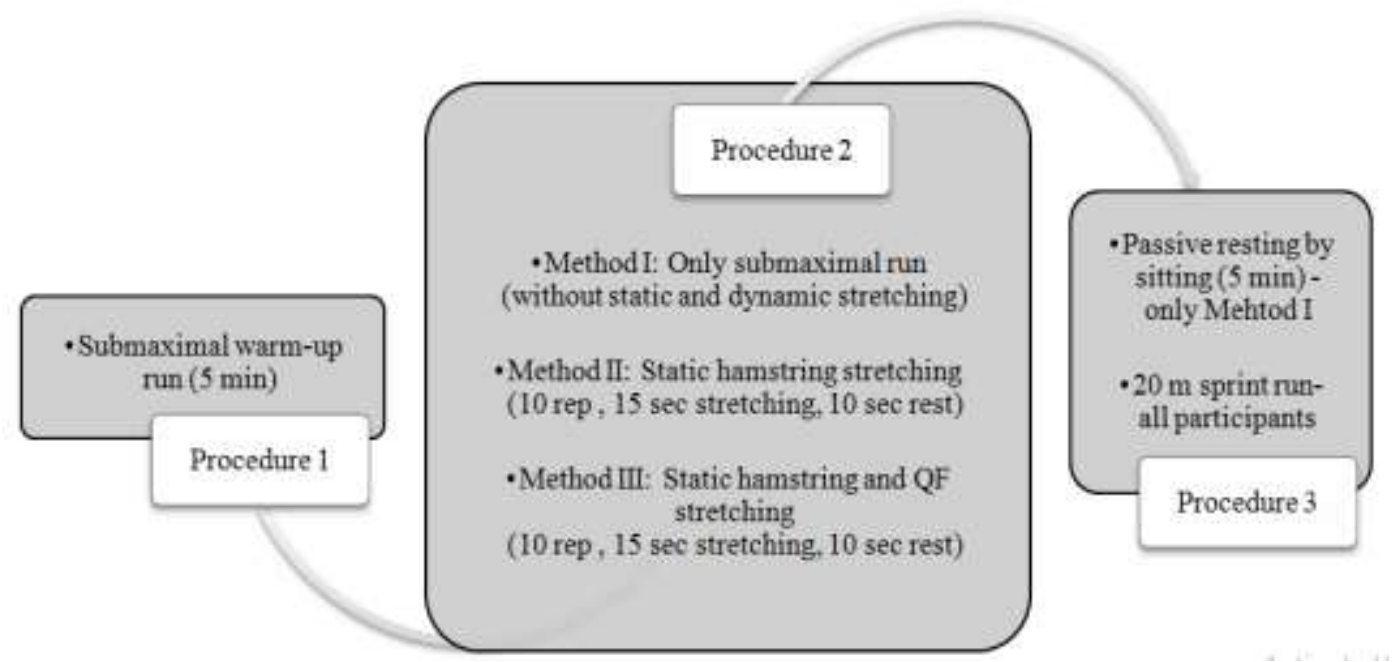

Fig 1: Summary of study design. min: minute, sec:second, QF: quadriceps femoris, m:meter.

\subsection{Statistical Method}

Descriptive statistics were used to describe the data. It was determined with the Skewness-Kurtosis test that the data were compatible with normal distribution. The 'Anova one-way analysis of variance' was conducted to determine whether there was a difference between the stretching protocols. The value of $p<0,05$ was accepted for statistical significance. All statistics were conducted with the SPSS 22.0 packagedsoftware. The data were presented as mean $(\mathrm{m}) \pm$ Standard Deviation $(\mathrm{SD})$.

\section{Results}

The average $( \pm \mathrm{SD})$ age of the participants was $22,9( \pm 1,3)$ years; the average height was $178,1( \pm 6,4)$ $\mathrm{cm}$; the average body weight was $77,7( \pm 12) \mathrm{kg}$. According to the data obtained as a result of the evaluation of sprint performances, when only the warm-up protocol (method I; mean \pm SD:3,54 $\pm 0,19 \mathrm{sec}$ ) was compared with the application, it was observed that passive stretching (method II; mean \pm SD:3,5 $\pm 0,16$ sec and method III; mean $\pm \mathrm{SD}: 3,49 \pm 0,16 \mathrm{sec}$ ) applied to the hamstring muscle had a numerically positive effect on the acute sprint performance. However, no statistically significant difference was found between Method I, II, and III (p: 0.46, F: 0.78). The results of the variance analysis of sprint performances obtained after static stretching exercises applied to different muscle groups are indicated in Graphic 1.

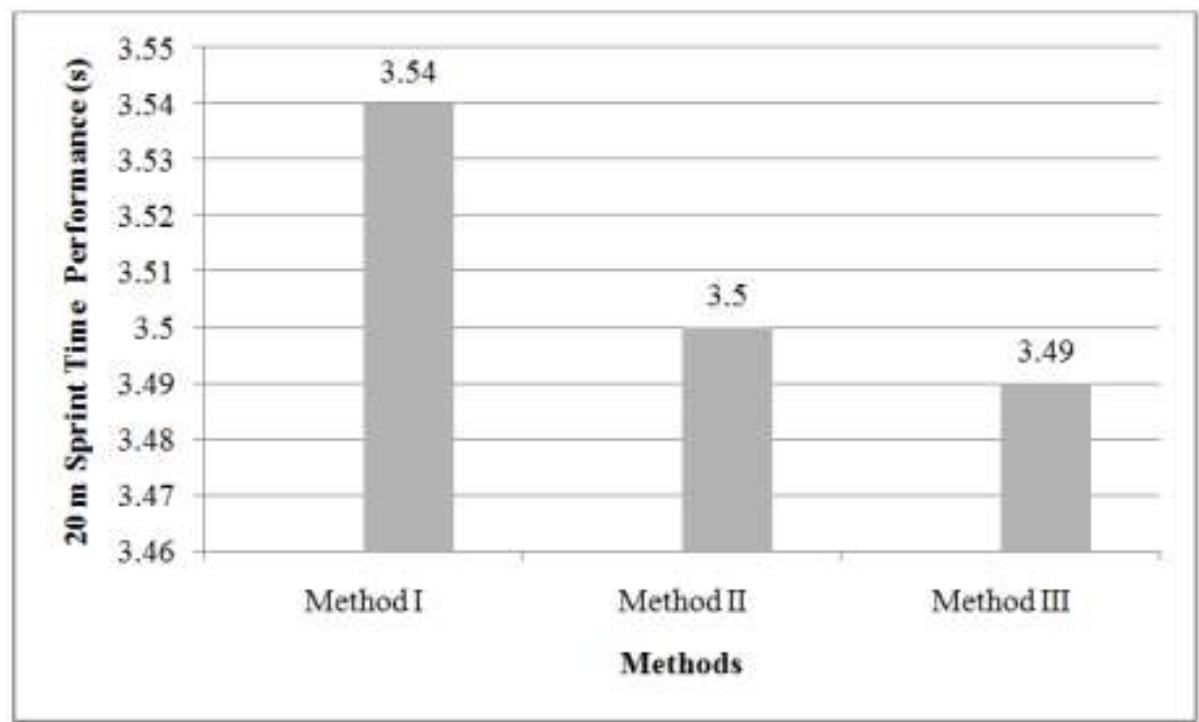

Graphic 1: Results of variance analysis of 20 meter sprint time performances (n:45).

The results are given on average. m: meters, s: seconds. Method I: only 5- minute warm-up run; Method II: 5- minute warm-up run and static hamstring stretching; Method III: 5- minute warm-up run, static hamstring and Quadriceps femoris stretching 


\section{Conclusion}

As a result of this study conducted to investigate the acute effect of static stretching applied to different muscle groups of the lower extremity, it was found out that although static stretching exhibited a statistically significant difference, it affected the 20-meter sprint performance positively. It is indisputable that achieving and sustaining the maximum performance following a structured training program is the ultimate goal of athletes [2]. The effect of stretching exercises, which are an indispensable part of training, on performance is considered to be among the subjects that should be enlightened by athletes and performers [2]. Thus, the researchers have structured their studies to find an answer to this question.

Although many studies can be found on this subject, it is known that no consensus was reached on the acute effects of static stretching exercises on the performance [3]. For example, Nelson et al. reported that when they applied static stretching to the bilateral extremity and unilateral extremity in front and back of the sprint running starts for $30 \mathrm{sec}$ and with 4 repetitions, all three methods caused lower performance when compared to the 20-m sprint running performed without stretching [13]. Stewart et al. stated that performing only warm-up running provides a higher performance increase when compared to stretching or stretching combined with warm-up running [4]. It was found out in a study focusing on the phases of sprint performance that static stretching has a negative effect on acceleration time, maximal velocity sprint time and total sprint time [9]. As a result of another study, while performing active or passive static stretching extends the 20-m sprint time, active dynamic stretching, on the contrary, reduces it [2]. In a similar study design, Taylor et al. reported that static stretching had negative effects on the vertical jumping height and $20-\mathrm{m}$ sprint time when compared to dynamic warm-up, on the other hand, when sport-specific skill-based warm-up was performed, the negative effects of static stretching disappeared [1].

While some of the researchers believe that static stretching exercises prevent the sportive performance, various stretching protocols containing different times and applied to different muscle groups continue to be used in the daily life for the protection from injuries [1]. In the light of recent studies, it is considered that the negative effect of static stretching on performance may be related to the duration of stretching exercise $[1,14]$. According to the recent data, passive stretching exercises in the 15-30 sec range were found to be more effective when compared to shorter or longer time stretching. More positive results were obtained especially when compared to static stretching and dynamic stretching applied to the hamstring muscle group [14]. Çoknaz et al. reported in their studies conducted with artistic gymnasts that the most appropriate duration for static stretching was $15 \mathrm{sec}$. Following the 15 -sec static stretching exercise with 10 repetitions, the joint range of motion increased significantly and the vertical jump test was not affected negatively [12]. Considering these data, it was observed during our study that the stretching protocols we created did not affect the 20-m sprint time performance negatively, moreover, they provided a numerical improvement.

Fortier et al. emphasized that performing static stretching with plyometric exercise combination did not affect sprint time and all protocols only reduced the vertical jump height whichever protocol was applied [15]. Christensen et al. concluded that using a miniband during the warm-up period caused a longer sprint time when compared to the protocol including static stretching; however, the best sprint time was achieved by the control group [16]. In a study in which the effects of different stretching types on the performance of football players were evaluated, similar results to our study were obtained in terms of the 20-m sprint time, however, the authors reported that dynamic stretching contributed more positively to the $10-\mathrm{m}$ acceleration time and zig zag skill when compared to static stretching [17]. Vetter found out that 6 different warm-up protocols containing different combinations of static stretching did not create differences in the sprint running performance, the only difference resulted from gender [18].

It was proved with various studies that the chronic application of static stretching did not affect sprint performance negatively [19]. It was indicated in these studies that chronic flexion and stretching exercises did not reduce athletic performance; on the contrary, they contributed to the performance increase. It is recommended that flexion and stretching exercises, which are indispensable parts of the warm-up and training program, should be certainly performed regularly during the training periods [19].

Our study is the first study evaluating the effect of static stretching applied to different muscle groups of the lower extremity. According to the data we obtained, the effect of static stretching applied only to the hamstring muscle or by combining the hamstring muscle and QF muscle on sprint time is similar. Moreover, although statistical significance cannot be obtained, it is positive when compared to the situation when stretching is not performed. Therefore, we think that short-term static stretching exercises should be performed before the competition, without the concern of performance loss.

\section{References}

[1]. K. L.Taylor, J. M. Sheppard, H.Lee, and N. Plummer, Negative effect of static stretching restored when combined with a sport specific warm-up component, Journal of Science and Medicine in Sport, 12(6), 2009, 657-661.

[2]. Y.Alikhajeh, N. M.Rahimi, K.Fazeli, and H.Fazeli, The effect of different warm up stretch protocols on 20m-sprint performance in trained soccer players. Procedia-Social and Behavioral Sciences, 46, 2012, 2210-2214. 
[3]. K.Mine, T. Nakayama, S.Milanese, and K.Grimmer, Acute effects of stretching on maximal muscle strength and functional performance: A systematic review of Japanese-language randomised controlled trials.,Manual therapy, 21, 2016, 54-62.

[4]. M.Stewart, R.Adams, A. Alonso, B. Van Koesveld, and S.Campbell, Warm-up or stretch as preparation for sprint performance?, Journal of Science and Medicine in Sport, 10(6), 2007, 403-410.

[5]. G. C. E.Silva, A.Silveira, J. Novaes, F. Di Masi, M. Conceicao, and E. Dantas, Acute effects of static and proprioceptive neuromuscular facilitation stretching on sprint performance in male swimmers, Med Sport, 67, 2014, 119-128.

[6]. G. Dar, Dynamic stretching during warm-up prior to running, Edorium J Sports Med 1, 2015, 7-9.

[7]. S. M.Marek, J. T.Cramer, A. L.Fincher, and L. L. Massey, Acute effects of static and proprioceptive neuromuscular facilitation stretching on muscle strength and power output, Journal of Athletic Training, 40(2), 2005, 94-103.

[8]. A. Zakas, G.Doganis, V.Papakonstandinou, T. Sentelidis, and E. Vamvakoudis, Acute effects of static stretching duration on isokinetic peak torque production of soccer players, Journal of Bodywork and Movement Therapies, 10(2), 2006, 89-95.

[9]. A. L.Sayers, R. S. Farley, D. K. Fuller, C. B. Jubenville, and J. L. Caputo, The effect of static stretching on phases of sprint performance in elite soccer players, The Journal of Strength and Conditioning Research, 22(5), 2008, 1416-1421.

[10]. F. G. Shellock, and W. E. Prentice, Warming-up and stretching for improved physical performance and prevention of sports-related injuries, Sports Medicine, 2(4), 1985, 267-278.

[11]. M. J. Alter, Science of Stretching, (Champaign, IL: Human Kinetics, 1988).

[12]. H. Çoknaz, N. Ü.Yıldırım and N. Özengin, Artistik Cimnastikçilerde Farklı Germe Sürelerinin Performansa Etkisi, SPORMETRE Beden Eğitimi ve Spor Bilimleri Dergisi, 3, 2008, 151-157.

[13]. A. G. Nelson, N. M. Driscoll, D. K. Landin, M. A.Young, and I. C. Schexnayder, Acute effects of passive muscle stretching on sprint performance, Journal of sports sciences, 23(5), 2005. 449-454.

[14]. S. B.Thacker, J. Gilchrist, D. F Stroup, and Jr, C. D Kimsey, The impact of stretching on sports injury risk: a systematic review of the literature, Medicine and Science in Sports and Exercise, 36(3), 2004, 371-378.

[15]. J. Fortier, G. Lattier, and N. Babault, Acute effects of short-duration isolated static stretching or combined with dynamic exercises on strength, jump and sprint performance, Science and Sports, 28(5), 2013, 111-117.

[16]. B. Christensen, R. Napoli, K. Hackney, J. Miller, and H. Murata, The effects of two different types of dynamic warm-up and statıc stretching on power and speed. In ISBS-Conference Proceedings Archive, 2016 November, 34(1).

[17]. T. Little, and A. G. Williams, Effects of differential stretching protocols during warm-ups on high-speed motor capacities in professional soccer players, The Journal of Strength and Conditioning Research, 20(1), 2006, 203-307.

[18]. R. E. Vetter, Effects of six warm-up protocols on sprint and jump performance, The Journal of Strength and Conditioning Research, 21(3), 2007, 819-823.

[19]. D. M.Bazett-Jones, M. H.Gibson, and J. M. McBride, Sprint and vertical jump performances are not affected by six weeks of static hamstring stretching, The Journal of Strength and Conditioning Research, 22 (1), 2008, 25-31. 